\title{
Anosmia and Ageusia in Covid-19 Patients
}

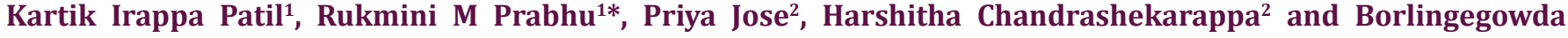 \\ Viswanatha $^{3}$
}

${ }^{1}$ Senior residents, Department of ENT, Bangalore Medical College \& Research Institute, India

${ }^{2}$ Postgraduate, Department of ENT, Bangalore Medical College \& Research Institute, India

${ }^{3}$ Professor and Head, Department of ENT, Bangalore Medical College \& Research Institute, India

*Corresponding author: Rukmini M Prabhu, Senior Resident, Department of ENT, Bangalore Medical College\& Research Institute, Bangalore, India

\begin{abstract}
Introduction: The Severe Acute Respiratory Syndrome Coronavirus 2(SARS CoV2), started in Wuhan in China at December 2019.The symptoms mainly included fatigue, fever, dry and productive cough, shortness of breath, chest compression, myalgia, diarrhea, vomiting, anorexia, headache, sore throat, dizziness, palpitations, and, chest pain. Anosmia and/or ageusia may present as a symptom alone in cases of COVID-19. Hence it is necessary to test or quarantine patients with these complaints.

Aims and objectives: To determine the prevalence of anosmia and ageusia in patients with COVID-19 presenting to our tertiary care center.

Materials and Methods: All patients with confirmed real time polymerase chain reaction (RT-PCR) positive testing for the SARS - CoV-2 viral genome was assessed for symptoms and signs of olfactory and taste disturbance.Patients were asked to quantify their loss of sense of smell and taste, subjectively into mild, moderate and severe.

Results: Out of 2000 patients 95 patients (4.75\%) had loss of sense of smell, and 143 patients (7.15\%) had taste disturbance. 85 patients (4.25\%) had both loss of smell and taste sensation. Out of 95 patients with loss of smell sensation, 25 patients (26.31\%) had associated comorbidities, 32 patients (22.37\%) out of the 143 with loss of taste sensation had comorbidities and 19(22.35\%) patients out of the 110 patients with loss of both sensations had associated comorbidities.Out of 95 patients with loss of smell sensation, $72(75.78 \%)$ patients recovered spontaneously. Out of 143 patients with loss of taste, 102 (79.44) patients recovered spontaneously. Among the patients with both symptoms, 59 (69.41\%) recovered spontaneously.

Conclusion: Loss of smell and taste sensation is common, especially in COVID-19 disease with mild to moderate symptoms, and it can appear without any other general and ENT-related symptoms associated with COVID-19 disease or it may be the only symptom of COVID-19 disease. During the COVID-19 pandemic, screening tests performed in patients with loss of taste and smell sensation due to suspected COVID-19 disease will allow early diagnosis and treatment of patients.
\end{abstract}

Keywords: COVID-19; anosmia; ageusia; prevalence

\section{Introduction}

The Severe Acute Respiratory Syndrome Coronavirus 2(SARS CoV2) started in Wuhan in China in December 2019[1]. Since then, the new virus, also known as Coronavirus Disease 2019 (COVID-19), has spread dramatically all over the world crossing all borders till the World Health Organization (WHO) confirmed it as a pandemic disease on March 11, 2020 [2].At the initial times of COVID-19 disease, in China, the symptoms mainly included fatigue, fever, dry and productive cough, shortness of breath, chest compression, myalgia, diarrhea, vomiting, anorexia, headache, sore throat, dizziness, palpitations, and, chest pain[3,4].Since then, there has been an increase in studies from different parts of the world about the significant increase in the cases number presenting with loss of smell alone[5].The pathophysiology of loss of smell developing after infection with this virus has been suggested to be 
due to olfactory epithelial damage or due to spread to the central nervous system[6,7].The behavior of SARS-CoV-2 is different in different patients. This prevents suspicion of the disease clinically, delays diagnosis and isolation of patients infected with the virus, make it difficult to deal with the disease[8].Anosmia and/or ageusia may present as symptom alone in cases of COVID-19. Hence it is necessary to test or a quarantine patient with this complaint. The aim of our study was to determine the prevalence of anosmia and ageusia in patients with COVID-19 presenting to our tertiary care center.

\section{Materials and Methods}

All patients with confirmed reverse transcriptase polymerase chain reaction (RT-PCR )-positive testing for the SARS -CoV-2 viral genome were assessed for symptoms and signs of olfactory and taste disturbance. 2000 patients who presented to our tertiary care designated COVID hospital were included in the study. Health workers with confirmed positive PCR test results were voluntarily enrolled in the study. All subjects provided informed consent to participate in the study. Patients were asked to quantify their loss of sense of smell and taste, subjectively into mild, moderate, and severe.

\section{The inclusion criteria were}

a) Patients $>18$ years of age with a positive COVID -19 test.

b) Patients having mild to moderate symptoms.

\section{The exclusion criteria were}

a) Age $<18$ years old,

b) No confirmed positive PCR test result

c) A history of chronic nasal problems

d) Recent head injury, recent nasal or ear surgery

e) Severe respiratory failure or treatment in the intensive care unit.

\section{Results}

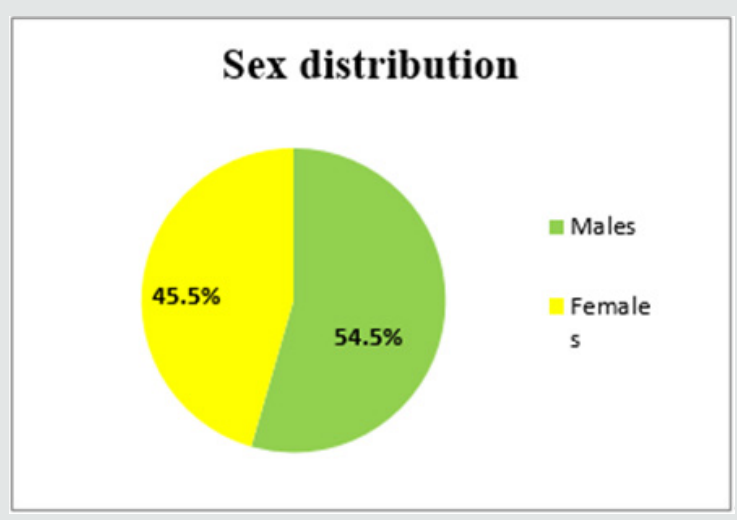

Figure 1: Showing the sex distribution of patients.
A total of 2000 patients were included in the study. The patients ranged in age from 18 to 70 years, including 1090 males (54.5\%) and 910 females (45.5\%) (Figure 1\& Table 1).The most prevalent comorbidities in these patients were (Figure $2 \&$ Table 2).

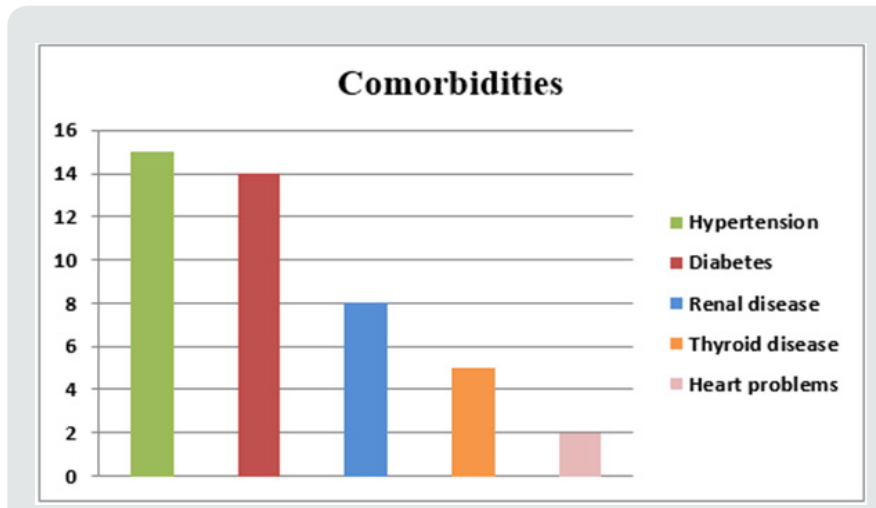

Figure 2: Showing various comorbidities seen in patients.

Table 1: Showing the sex distribution of patients.

\begin{tabular}{|c|c|c|}
\hline & No. of Patients & Percentage\% \\
\hline Male & 1090 & 54.5 \\
\hline Female & 910 & 45.5 \\
\hline
\end{tabular}

Table 2: Showing comorbidities seen in patients.

\begin{tabular}{|c|c|c|}
\hline Comorbidities & No. of Patients $(\mathbf{n}=\mathbf{2 0 0 0})$ & Percentage \% \\
\hline Hypertension & 300 & 15 \\
\hline Diabetes & 280 & 14 \\
\hline Renal disease & 160 & 8 \\
\hline Thyroid disease & 100 & 5 \\
\hline Heart problems & 40 & 2 \\
\hline Total & 880 & 44 \\
\hline
\end{tabular}

a) High blood pressure in 15\%,

b) Diabetes in $14 \%$,

c) Renal disease in $8 \%$,

d) Thyroid diseases in 5\%,

e) Heart problems in $2 \%$.

Out of 2000 patients 95 patients (4.75\%) had loss of sense of smell, and 143 patients $(7.15 \%)$ had taste disturbance. 85 patients $(4.25 \%)$ had both loss of smell and taste sensation(Figure 3 \&Table 3). Out of 95 patients with loss of smell sensation, 25 patients (26.31\%) had associated comorbidities, 32 patients $(22.37 \%)$ out of the 143 with loss of taste sensation had comorbidities and 19 $(22.35 \%)$ patients out of the 110 patients with loss of both sensations had associated comorbidities. It can be observed that most patients with olfactory and taste disturbances did not have comorbidities (Figure 4\& Table 4).Out of 95 patients with loss of smell sensation, 52 patients (54.73\%) had mild loss, 24 (25.27\%) pa- 
tients had moderate loss and $19(20 \%)$ patients had severe loss. Out of 143 patients with loss of taste, 80 (55.94\%) patients had mild loss, 43 (30.06\%) patients had moderate loss and 20 (13.98\%) patients had severe loss(Figure $5 \&$ Table 5).Patients were followed up for 15 days for improvement of symptoms. Out of 95 patients with loss of smell sensation, 72 (75.78\%) patients recovered spontaneously. Out of 143 patients with loss of taste, 102 (79.44\%) patients recovered spontaneously. Among the patients with both symptoms, 59 (69.41\%) recovered spontaneously(Figure 6\& Table 6).

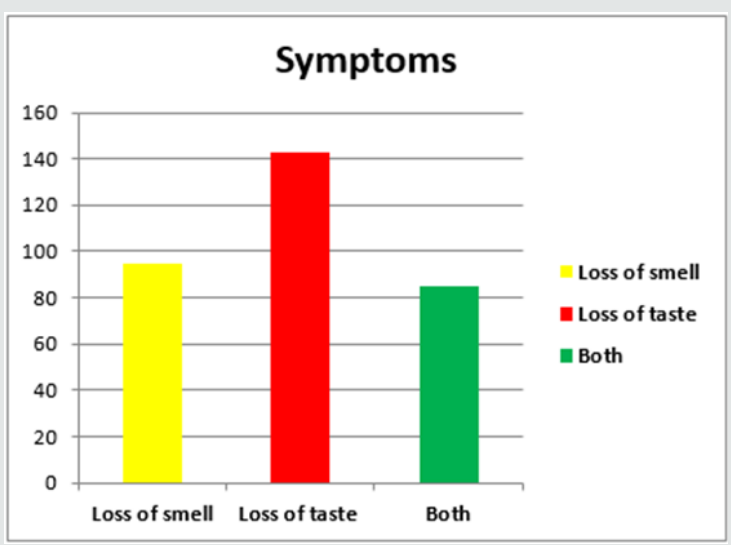

Figure 3: Showing patients with olfactory and taste disturbances.

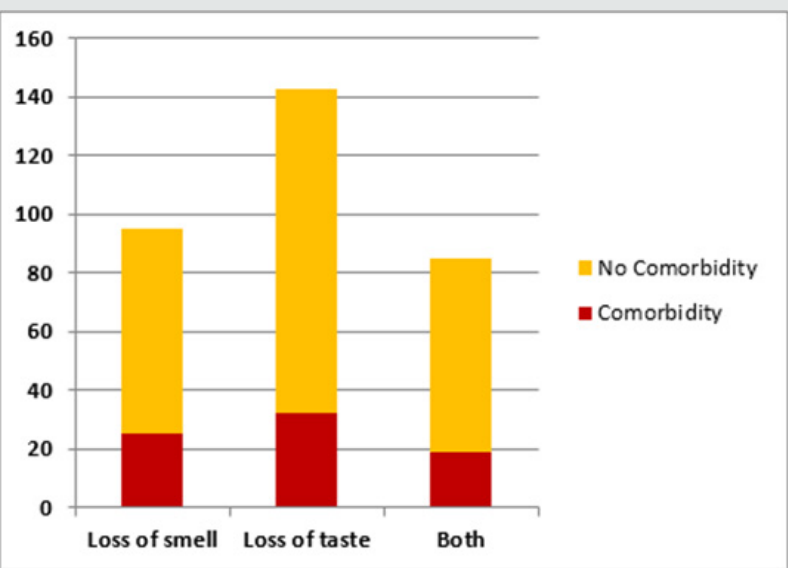

Figure 4: Showing symptoms in patients with comorbidity.

Table 3: Showing patients with symptoms.

\begin{tabular}{|c|c|}
\hline Symptoms & No. of Patients \\
\hline Only loss of smell & $95(4.75 \%)$ \\
\hline Only loss of taste & $143(7.15 \%)$ \\
\hline Both & $85(4.25 \%)$ \\
\hline
\end{tabular}

Table 4: Showing symptoms in patients with comorbidity.

\begin{tabular}{|c|c|c|}
\hline Symptoms & Comorbidity & No comorbidity \\
\hline Loss of smell & $25(26.31 \%)$ & $70(73.69 \%)$ \\
\hline
\end{tabular}

\begin{tabular}{|c|c|c|}
\hline Loss of taste & $32(22.37 \%)$ & $111(77.63 \%)$ \\
\hline Both & $19(22.35 \%)$ & $66(77.65 \%)$ \\
\hline
\end{tabular}

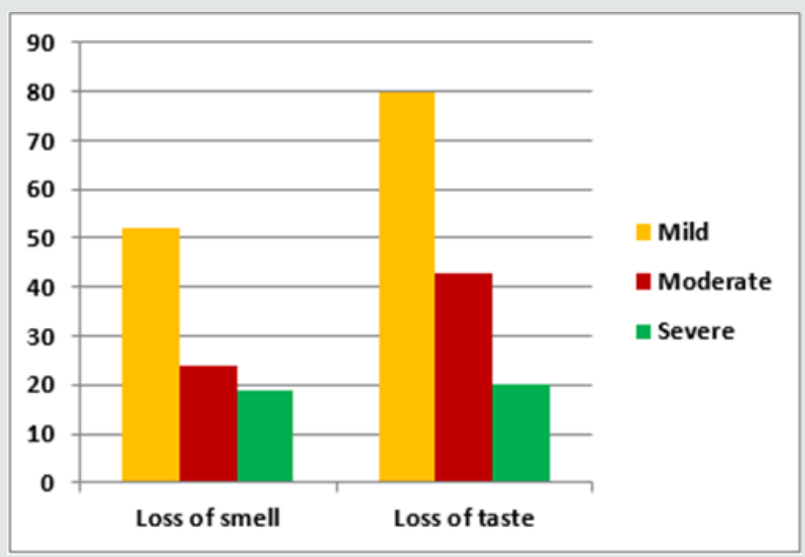

Figure 5: Showing severity of symptoms in patients.

Table 5: Showing severity of symptoms in patients.

\begin{tabular}{|c|c|c|c|c|}
\hline Symptoms & Mild & Moderate & Severe & Total \\
\hline Loss of smell & 52 & 24 & 19 & 95 \\
\hline Loss of taste & 80 & 43 & 20 & 143 \\
\hline
\end{tabular}

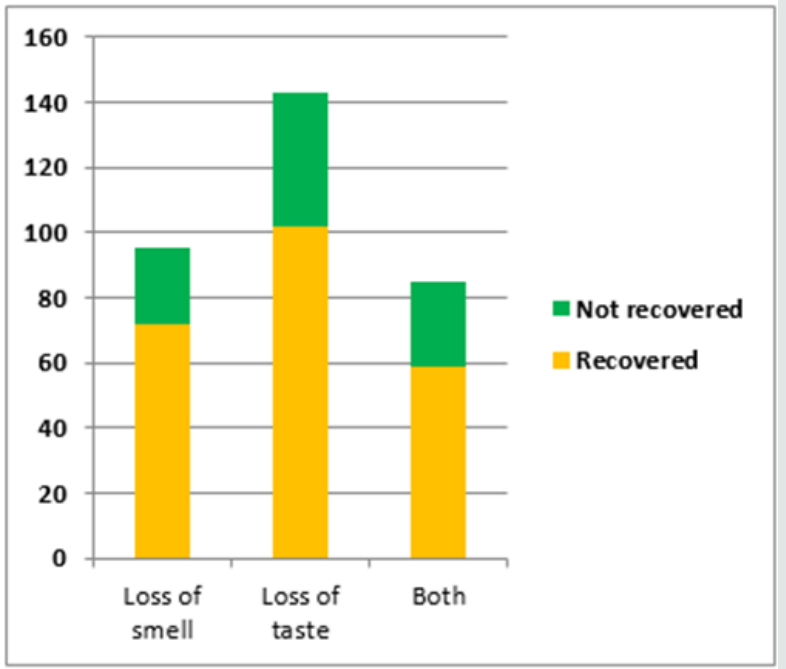

Figure 6: Showing patients who spontaneously recovered after 15 days.

Table 6: Showing patients who spontaneously recovered after 15 days.

\begin{tabular}{|c|c|c|c|}
\hline & Recovered & Not recovered & Total \\
\hline Loss of smell & $72(75.78 \%)$ & $23(24.22 \%)$ & 95 \\
\hline Loss of taste & $102(79.44 \%)$ & $41(20.56 \%)$ & 143 \\
\hline Both & $59(69.41 \%)$ & $26(30.59 \%)$ & 85 \\
\hline
\end{tabular}




\section{Discussion}

In December 2019, an epidemic,caused by the severe acute respiratory syndrome coronavirus 2, emerged from China [9]. COVID-19 can manifest with a wide clinical spectrum that ranges from no symptoms to septic shock and multi-organs dysfunction[10].Chemosensory dysfunctions are known to be common problems during the COVID-19 pandemic [5]. In a study conducted by Qiu et al, smell and taste abnormalities were the first and the only complaints in $10 \%$ of the subjects, and $25 \%$ of the children had only smell and taste abnormalities at the time of the presentation [11]. Therefore, these disorders can be considered as early indicators of COVID-19 disease and is necessary for screening and control of infection. In our study, we have investigated the frequency and severity of olfactory and taste related symptoms in patients with confirmed COVID-19 disease, the association with coexisting comorbidities,and the recovery process of the smell and taste disturbances.The first report that included loss of smell and taste sensation caused by SARS-CoV-2 was of a study conducted by Mao et al. which emphasized the neurological symptoms of the disease. They stated that approximately $6 \%$ of patients infected with SARS-CoV-2 had loss of sense of smell and that loss of sense of smell may be the first symptom before onset of other neurological symptoms [12]. In the study conducted by Al-Ani R et al, the prevalence rates of ageusia and anosmia, ageusia, and anosmia were $8.51 \%, 11.35 \%$, and $4.96 \%$, respectively [5]. Paderno et al. in their study observed that the olfactory and gustatory dysfunctions were seen in $83 \%$ and $89 \%$ of patients, respectively. Tong et al. [14] in a systematic review and meta-analysis study [13], observed that the prevalence rate of the 10 investigations which analyzed the olfactory dysfunction in patients with COVID-19 disease was $52.73 \%$, whereas nine investigations reported that the prevalence rate of the gustatory dysfunction was $43.93 \%$.Al-Ani $\mathrm{R}$ et al. [15] postulated that this variability among various studies might be attributed to the variation in the ethnicity, sample size, and type of the sample[5].In our study, out of 2000 patients 95 patients (4.75\%) had loss of sense of smell, and 143 patients (7.15\%) had taste disturbance. 85 patients (4.25\%) had both loss of smell and taste sensation.

In a study conducted by Kaye R et al [15], utilizing the COVID-19 Anosmia Reporting Tool developed by the American Academy of Otolaryngology-Head and Neck Surgery for clinicians indicated that $73 \%$ of 237 patients had loss of sense of smell at admission to hospital, and $26.6 \%$ of these patients had loss of sense of smell as the main complaint at the time of admission to hospital. They reported that $85 \%$ of the patients who described loss of sense of smell recovered within the first 10 days, and the average recovery time was 7.2 days.Lechien JR et al. [16] reported that $67.8 \%$ of their patients with loss of sense of smell and $78.9 \%$ of patients with loss of sense of taste recovered to various degrees within an average of 8 days [16]. In our study, patients were followed up for 15 days for improvement of symptoms. We found that $75.78 \%$ patients with loss of smell sensation, $79.44 \%$ with loss of taste sensation and $69.4 \%$ of patients with both symptoms recovered at 15 days. In a study conducted by Sakalli E et al, out of 172 patients, 88 patients were reported to have loss of sense of smell related to SARS-CoV-2 infection. Among them, loss of sense of smell was mild in one (1.2\%) patient, moderate in 24 (14\%), and severe in $62(36.0 \%)$ patients[17]. We found that out of 95 patients with loss of smell sensation, 52 patients (54.73\%) had mild loss, 24 (25.27\%) patients had moderate loss and $19(20 \%)$ patients had severe loss. Out of 143 patients with loss of taste, 80 (55.94\%) patients had mild loss, $43(30.06 \%)$ patients had moderate loss and 20 (13.98\%) patients had severe loss. It was also observed in our study that most patients with olfactory and taste disturbances did not have any associated comorbidities.There are no proven mechanisms for the loss of smell and taste sensation. There is no prior investigation that studied the possible association between coronavirus and the appearance of taste alteration. Zhou et al. [18] have been confirmed that COVID-19 uses the cellular angiotensin-converting enzyme 2[18].It is possible that the COVID-19 causes taste dysfunction similar to that caused by the ACE2 inhibitors[19]. Though the mechanisms for the smell and taste loss in COVID-19 disease are unclear, we observed that SARS-CoV-2 does cause loss of smell and taste sensation in infected patients, and that majority of patients recover spontaneously.The limitations of our study include the following:

a) Subjective assessment was done, and objective diagnostic tests were not used to evaluate the severity and frequency of the STL symptoms and improvement of STL.

b) We did not include patients with severe symptoms and those treated in the intensive care unit. Therefore, our study population might not be representative of all patients with COVID-19 disease.

c) Patients were followed up for 15 days for assessment of recovery of smell and taste sensation, therefore we do not have long term follow-up results for patients who did not recover within 15 days.

\section{Conclusion}

Loss of smell and taste sensation is common, especially in COVID-19 disease with mild to moderate symptoms, and it can appear without any other general and ENT-related symptoms associated with COVID-19 disease or it may be the only symptom of COVID-19 disease. During the COVID-19 pandemic, screening tests performed in patients with loss of taste and smell sensation due to suspected COVID-19 disease will allow early diagnosis and treatment of patients. 


\section{References}

1. Xia W, Shao J , Guo Y , Peng X, Li Z , Hu D (2020) Clinical and CT features in pediatric patients with COVID-19 infection: different points from adults. Pediatr Pulmonol 55(5): 1169-1174.

2. Cucinotta D, Vanelli M (2020) WHO declares COVID-19 a pandemic. Actabio-medica: Atenei Parmensis 91(1): 157-160.

3. Z Wu, JM McGoogan (2020) Characteristics of and important lessons from the coronavirus disease 2019 (COVID-19) outbreak in China: summary of a report of 72314 cases from the Chinese Center for Disease Control and Prevention. Jama 323(13): 1239-1242.

4. Wang Z, Yang B, Li Q, Wen L, Zhang R (2020) Clinical Features of 69 Cases with Coronavirus Disease 2019 in Wuhan, China. Clinical Infectious Diseases 71(15): 769-777.

5. Al-Ani R, Acharya D (2020) Prevalence of Anosmia and Ageusia in Patients with COVID-19 at a Primary Health Center, Doha, Qatar. Indian Journal of Otolaryngology and Head \& Neck Surgery 19: 1-7.

6. Suzuki M, Saito K, Min WP, Vladau C, Toida K, et al. (2007) Identification of viruses in patients with postviral olfactory dysfunction. Laryngoscope 117(2): 272-277.

7. Van Riel D, Verdijk R, Kuiken T (2015) The olfactory nerve: a shortcut for influenza and other viral diseases into the central nervous system. J Pathol 235(2): 277-287.

8. Sakalli E, Temirbekov D, Bayri E, Alis E, Erdurak S, et al. (2020) Ear nose throat-related symptoms with a focus on loss of smell and/or taste in COVID-19 patients. American Journal of Otolaryngology 41(6): 102622

9. Guan WJ, Ni ZY, Hu Y, Liag WH, Ou CQ, et al. (2020) Clinical characteristics of coronavirus disease 2019 in China. N Engl J Med 382(18): 1708 -1720.

10. Cascella M, Rajnik M, Cuomo A, Dulebohn SC, Di Napoli R (2020) Features evaluation and treatment coronavirus (COVID-19). In Stat pearls Stat Pearls Publishing.
11. Qiu C, Cui C, Hautefort C, Haehner A, Zhao J, et al. (2020) Olfactory and Gustatory Dysfunction as an Early Identifier of COVID-19 in Adults and Children: An International Multicenter Study. Otolaryngology-Head and Neck Surgery 163(4): 714-721.

12. Mao L, Jin H, Wang M (2020) Hospitalized Patients with Coronavirus Disease 2019 in Wuhan, China. JAMA Neurol.

13. Paderno A, Mattavelli D, Rampinelli V, Grammatica A, Raffetti E, et al (2020) Olfactory and Gustatory Outcomes in COVID-19: A Prospective Evaluation in Nonhospitalized Subjects. Otolaryngology-Head and Neck Surgery.

14. Tong J, Wong A, Zhu D, Fastenberg J, Tham T (2020) The Prevalence of Olfactory and Gustatory Dysfunction in COVID-19 Patients: A Systematic Review and Meta-analysis. Otolaryngology-Head and Neck Surgery 163(1): 3-11.

15. Kaye R, Chang C, Kazahaya K, Brereton J, Denneny J (2020) COVID-19 Anosmia Reporting Tool: Initial Findings. Otolaryngology-Head and Neck Surgery 163(1): 132-134

16. Lechien JR, ChiesaEstomba CM, De Siati DR (2020) Olfactory and gustatory dysfunctions as a clinical presentation of mild- to- moderate forms of the coronavirus disease (COVID- 19):a multicenter European study. Eur Arch Otorhinolaryngol 277(8): 2251-2261.

17. RM Al-Ani RM (2020) Smell and Taste Abnormalities due to COVID-19 Al- Anbar Med J 16(1): 1-2.

18. P Zhou (2020) A pneumonia outbreak associated with a new coronavirus of probable bat origin. Nature 579(7798): 270-273.

19.J Suliburska, G Duda, D PupekMusialik (2012) The influence of hypotensive drugs on the taste sensitivity in patients with primary hypertension. Acta Pol Pharm 69(1): 121-127.

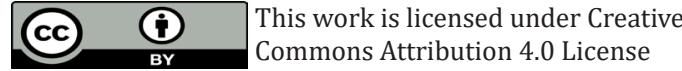

To Submit Your Article Click Here: Submit Article

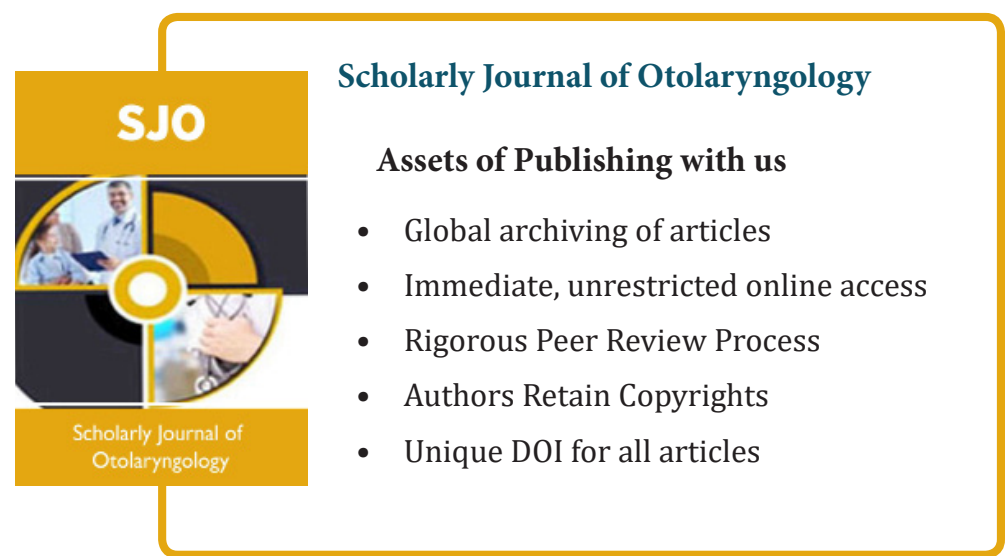

